\title{
Plan for Prevention of Natural Hazards in Urban Areas. Case of the City of Constantine (Algeria)
}

\author{
Ykhlef Boubakeur $^{1}$, Belouar Abdelghani ${ }^{1}$ and Boubidi Sayed ${ }^{1}$ \\ ${ }^{1}$ University Constantine 1 LMDC, Department of civil Engineering, Constantine, Algeria
}

\begin{abstract}
Natural hazards are caused by different phenomena: landslides, earthquakes, floods etc. All the manifestations of forces of nature are called phenomena. We are speaking then of natural risks when these problems are threatening, with varying degrees, human activities, causing significant damage to human life, property and the environment. The need to consider natural hazards in land use planning tasks has become a major concern. During these past years, Algeria has been hit by frequent natural disasters, with the most recent ones endangering the lives of people and causing priceless damage, faced with such a situation the company of adequate measures, capable of exercising effective prevention, is essential. In addition to the seismic risk, for which prevention still needs to be improved, Algeria must also face gravity processes such as landslides. By their suddenness, they can put people in danger and destroy entire buildings involving the evacuation of entire neighbourhoods where the financial impact is significant on the state budget and local government. The main interest of this paper is the feasibility of a plan for prevention of natural disasters related to landslides based on geological maps, topography, hydrogeological and on existing buildings and vulnerability, and eventually lead to a Zoning risk that would be considered for inclusion in the Master Plan of Urban Planning and Land Use Plan and provide support for decisions taken by local authorities for the selection of sites.
\end{abstract}

\section{Introduction et historique des plans de préventions des risques}

Le risque de glissement est un événement spatial, lié au terrain, et la carte est toujours son support privilégié. La cartographie et la base de données tentent de répondre aux questions suivantes :

- Où peut se produire un glissement de terrain?

- Quand et à quelle vitesse cela arrivera-t-il ?

- Quel type de mouvement?

- Quelle est la marge de sécurité ?

- Quelles seront les dimensions du glissement?

- Quelle sera son extension (aval ou amont)?

Les études cartographiques sont faites à différentes échelles, les études à grande échelle étant du domaine du pouvoir local. On peut distinguer trois classes d'échelle : inférieure à $1 / 100000$, supérieure à $1 / 10000$ et entre ces deux limites (Vaunat 1997) [1]. En France les échelles employées sont le 1/25000 et le 1/5000. (Carte d'état major et cadastre) (Leroi 1996) [2]. En Norvège l'état a un rôle de prévention, il répertorie les risques et formule des conseils. Il effectue des travaux de prévention, les études détaillées sont à la charge des municipalités ainsi que les travaux de réfection. Les échelles employées sont le $1 / 25000$ et le $1 / 5000$ (Berggren 1992) [3]. En Suède, l'Etat étudie le risque à l'échelle du territoire et les municipalités assument la responsabilité de la reconnaissance détaillée. Le gouvernement accorde une aide si le risque est imminent (Ahlberg et al 1988) [4]. A Hong-kong, (200 îles, 1050 km2), le Geotechnical Control Office fait l'analyse des risques relatifs au sol et fortement liés à la pluviométrie. Un réseau de stations pluviométriques très dense est l'élément essentiel du dispositif d'alerte. De nombreuses études statistiques ont permis d'établir une carte de classification des terrains, reliée à l'utilisation des terrains et aux limitations attachées à leur usage. De nombreuses analyses de stabilité sont aussi cartographiées. Les échelles employées sont le 1/20000 et le 1/2500 (Brand 1988) [5], (Fell et al 1996) [5]. En Italie, plusieurs programmes nationaux ont été entrepris pour cartographier tous les mouvements de terrain, soit par région soit par itinéraire.

Les échelles employées sont le 1/400000, 1/100000 et le $1 / 10000$. Dès 1908 , un programme avait recensé toutes les villes à reconstruire, programme qui n'a pas eu beaucoup de suites (Canutti 1988) [6]. Au Québec, la loi impose la réalisation de cartes de risques, qui sont importants vu la nature géologique des sols (argiles sensibles). Au Brésil, la ville de Rio de Janeiro, possède un inventaire cartographié des glissements de terrain ainsi que des cartes de risque (Barros 1992) [7]. En Roumanie, une approche multicritère a permis la mise en cuvre d'une cartographie grâce à un SIG (Rosenbaum et al 1996) [8]. Du fait des lois existant pour la plupart des pays, et de l'impulsion donnée par l'UNESCO, (décennie

This is an Open Access article distributed under the terms of the Creative Commons Attribution License 2.0, which permits unrestricted use, distribution, and reproduction in any medium, provided the original work is properly cited. 
des risques naturels), la nécessité de gestion et de décision s'impose dans presque partout. Le gestionnaire $\mathrm{du}$ territoire ne peut plus se contenter de descriptions et veut des outils de plus en plus performants pour pouvoir prendre des décisions pertinentes et équitables. En Algérie, la croissance galopante de la démographie, l'urbanisation anarchique, la dégradation de l'environnement et tous les dérèglements des écosystèmes font aggraver l'ampleur et la fréquence des catastrophes souligne "Symbiose" dans sa dernière édition.

Selon le Plan des Nations Unies pour l'Environnement, le nombre moyen de victimes est 150 fois plus élevé dans les pays en voie de développement que dans ceux développés. La revue spécialisée en Environnement estime que pour le cas de l'Algérie, il est plus qu'urgent de passer «d'une stratégie de lutte ou de réhabilitation à une stratégie de prévention des catastrophes »."Symbiose" se réfère à un récent colloque sur la prévention des risques pour citer le ministre de l'Aménagement du territoire et de l'environnement, Chérif Rahmani, qui a insisté sur une meilleure sensibilisation du public aux risques causés par les dangers environnementaux, naturels et technologiques. Il est à noter que la pollution par les hydrocarbures est un thème qui a particulièrement retenu l'attention des spécialistes.

En trois années, l'Algérie a été ébranlée par les inondations du quartier de Bab El Oued d'Alger en 2001; le tremblement de terre de Zemmouri-Boumerdès en 2003 et l'accident chimique (gazeux) survenu dans la zone industrielle de Skikda en 2004 et les glissements de terrains de Constantine 2005.Les pouvoirs publics sont encore fréquemment démunis en matière de prévision, de prévention et de gestion de phénomènes naturels aux conséquences parfois dramatiques (glissements de terrain, coulées boueuses, effondrements de falaises, chutes de blocs, effondrements de cavités souterraines etc.) L'opération vise à améliorer la connaissance acquise depuis plusieurs années dans les différents aspects de l'ingénierie des risques naturels: identification, caractérisation, prévision du comportement des phénomènes, définition des outils de cartographie et de gestion des risques liés aux mouvements de terrain au regard des personnes, des ouvrages, des réseaux et des biens menacés. D'une manière générale le risque majeur se caractérise par de nombreuses victimes, un coût important de dégâts matériels, des impacts sur l'environnement : la vulnérabilité mesure ces conséquences.

Le risque majeur est donc la confrontation d'un aléa avec des enjeux. Ainsi la société comme l'individu doivent s'organiser pour y faire face. Un événement potentiellement dangereux aléa n'est un risque majeur que s'il s'applique à une zone où des enjeux humains, économiques ou environnementaux sont en présence.

Deux critères caractérisent le risque majeur :

- une faible fréquence : l'homme et la société peuvent être d'autant plus enclins à l'ignorer que les catastrophes sont peu fréquentes ;

- une énorme gravité : nombreuses victimes, dommages importants aux biens et à l'environnement.
- Aléa : probabilité d'apparition d'un phénomène donné sur un territoire donné, dans une période de référence donnée. L'aléa est caractérisé par (3) trois éléments selon le cas, comme par exemple : description du phénomène, définition spatiale (point de départ et épandage) et appréciation de l'occurrence temporelle (rupture peu probable dans les 5 ans).

-Enjeu : Personnes, biens, équipements ou environnement menacés par l'aléa et susceptibles de subir des préjudices ou des dommages.

Tout citoyen a droit à l'information sur les risques auxquels il est soumis, ainsi que sur les moyens de s'en protéger.

Le terme risque comprend les risques de toute nature y compris les risques naturels. Le plan de prévention des risques approuvé vaut servitude d'utilité publique et impose des limites au droit de propriété et au droit d'utiliser le sol (Code de l'urbanisme); le PPR s'impose également aux POS (Plans d'occupation des sols) auquel il doit être annexé, et intervient dans la délivrance de permis de construire.

\section{Localisation du site cas de la ville de constantine (Algérie)}

\subsection{Historique}

Depuis une trentaine d'années, la ville de Constantine (Est de l'Algérie), située sur des terrains très escarpés, accidentés et sismiquement actifs, a connu épisodiquement de graves problèmes de glissements. Ces derniers se sont manifestés parallèlement à une urbanisation rapide et anarchique autour du site de l'ancienne ville. Ces mouvements se sont aggravés lors des pluies orageuses de 1986-1987, de juillet août 1994 et septembre 1998. En effet, la plupart des glissements de terrains sont réactivés au cours des hivers où la pluviométrie moyenne varie de $306 \mathrm{~mm}$ à $733 \mathrm{~mm}$.

De plus, la vétusté des réseaux enterrés de l'assainissement et de l'eau potable a subit des dommages, non localisés, ayant entraîné des fuites qui favorisent la lubrification des surfaces de décollement, aggravant de cette manière le problème.

Depuis 1998, des expertises effectuées par des spécialistes internationaux et nationaux sur l'ensemble des sites ont débouché sur des conclusions recommandant des études très ciblées sur chaque site afin de stabiliser, quand cela est possible, les mouvements du sol et d'instrumenter chaque zone à des fins de prévention (Paulsen et al 1998) [9].

Nous présentons dans cet article, les différents sites touchés par ce phénomène, d'une part, et possédant une banque de données importante et d'autre part, certains représentent des cas typiques de glissements anthropiques suite à une urbanisation rapide et anarchique.

En 1999, sur l'ensemble de la zone urbaine (ancien et nouveau tissu urbain), seize (16) sites présentant un fort potentiel de glissement ont été recensés.

La zone urbaine de Constantine d'une superficie de 5001 ha présente dix zones de glissements (figure1) 
représentant une surface totale de 165 ha soit 3,3\% avec une grande concentration d'habitants de l'ordre de $16 \%$.

Les glissements de terrains qui guettent la ville des ponts et lui font encourir un danger réel et imminent d'une catastrophe à grande échelle. Ces mouvements sont très anciens et se réactivent quand l'occasion se présente qui sont dans la figure 2. La ville de Constantine, objet du présent article, est relativement bien documentée. Les objectifs de ce travail sont :

- la synthèse des données de cette ville.

-l'évaluation des caractéristiques géologiques, topographiques, hydrogéologique et géotechniques et la vulnérabilité du bâti de cette ville.

- le développement d'un modèle de référence par rapport aux zones voisines.

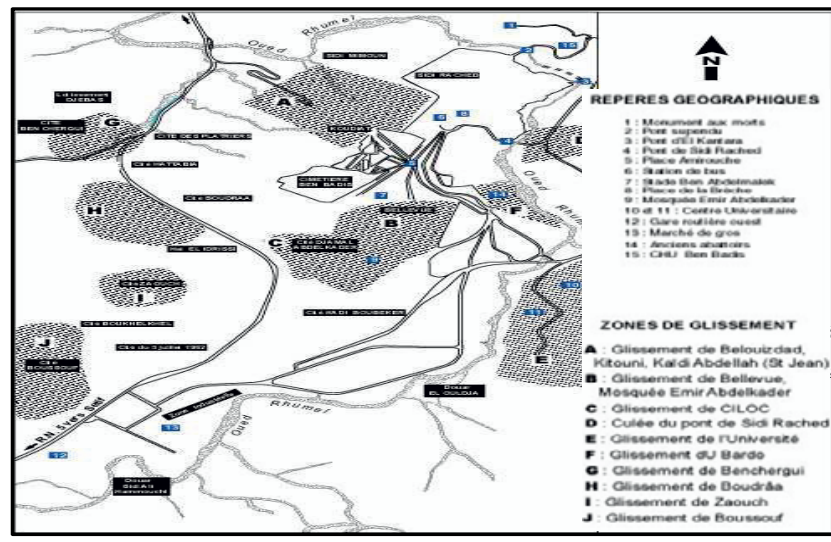

Fig. 1. Principales zones de glissements localisées dans le périmètre urbain de la ville de Constantine (Algérie). (Belouar 2005) [10].

\subsection{Géologie}

Les unités géologiques (figure 3) qui affleurent dans la région sont :

- Le rocher de Constantine,

- Les Mio-pliocène marneux,

- Les dépôts récents du Quaternaire et les matériaux de remblai disposés par l'activité humaine.

Les deux dernières unités, peu ou pas consolidées, sont à l'origine des glissements de Constantine.

Le rocher de Constantine: Ce sont des calcaires massifs d'âge Cénomanien à Turonien sur lesquels l'ancienne ville a été construite. Tectoniquement ce rocher se présente comme un horst faillé à flanc SE monoclinal ayant continué à se soulever inégalement en "touches de piano" pendant le Quaternaire.

Les marnes Mio-pliocène: Cette série calcaire est surmontée par une épaisse couche de marnes noires d'âge Campanien présentant parfois une faible schistosité. Quant la pente du substratum sur lequel repose ces marnes est importante des glissements se manifestent à la faveur de phénomènes naturels exceptionnels tels par exemple les pluies diluviennes et le tremblement de terre de 1910 et 1947 respectivement qui ont entraînés des sérieux dommages à la culée Est du Pont de Sidi Rached. Ces unités affleurent sur le côté Est du Rhumel. Le Nord de la confluence Boumerzoug et au Sud du Rocher y compris la partie supérieure de la culée Est du Pont Sidi Rached. Les conglomérats et sables du Quaternaire:
Parallèlement aux mouvements tectoniques de soulèvement du rocher, une puissante série de couches continentales se déposait. Il s'agit de haut en bas d'une alternance de conglomérats et d'argiles sableuses rouges : - Des cailloutis avec blocs pouvant atteindre $0,5 \mathrm{~m} 3$ de volume noyés dans une argile limoneuse d'argile sableuse : Epaisseur 0 à $3 \mathrm{~m}$.

- Un ensemble plus compact de calcaires lacustres, travertins, calcaires pisolithiques et sables passant à limons et vases présentant des débris de Vertébrés associés à une industrie paléolitique. Epaisseur 0 à $10 \mathrm{~m}$. - Placage d'argiles rouges de décalcification. Epaisseur 0 à $1 \mathrm{~m}$.

- Des dépôts alluvionnaires formés de sables et de limons sont localisés au niveau du lit du Rhummel. Leur épaisseur est variable.

- Les remblais issus de l'activité humaine sont très répandus dans la région. Un grand remblai existe près de la terminaison Ouest du Pont Sidi Rached. Les mouvements de sol constatés sur ces niveaux ont été la cause des dommages qui affectent les constructions et les routes avoisinantes : la station des bus Krikri en est un exemple. Plus grave encore, une ex- décharge d'ordures ménagères, pose un risque considérable aux maisons construites en amont et à l'aval de celle-ci.

L'ensemble de ces formations continentales, peu ou pas consolidées, sont très sensible aux instabilités des pentes et aux glissements de terrain (Deleau1967, Vila 1977, Lahondere 1987) [11-12-13].

\subsection{Morphologie}

La ville de Constantine s'est développée sur une topographie très escarpée et accidentée du Tell Constantinois. Le site est dominé par le rocher de Constantine qui se présente comme un escarpement calcaire avec une rive Ouest abrupte. L'altitude du rocher est approximativement $625 \mathrm{~m}$. En s'écoulant vers le Nord. L'oued Rhumel coupe le rocher en une gorge spectaculaire formée par karstification (figure4). Le Rhumel au pont d'EI Milia atteint environ $360 \mathrm{~m}$. créant une dénivelé maximum de $265 \mathrm{~m}$ dans la région de notre étude.

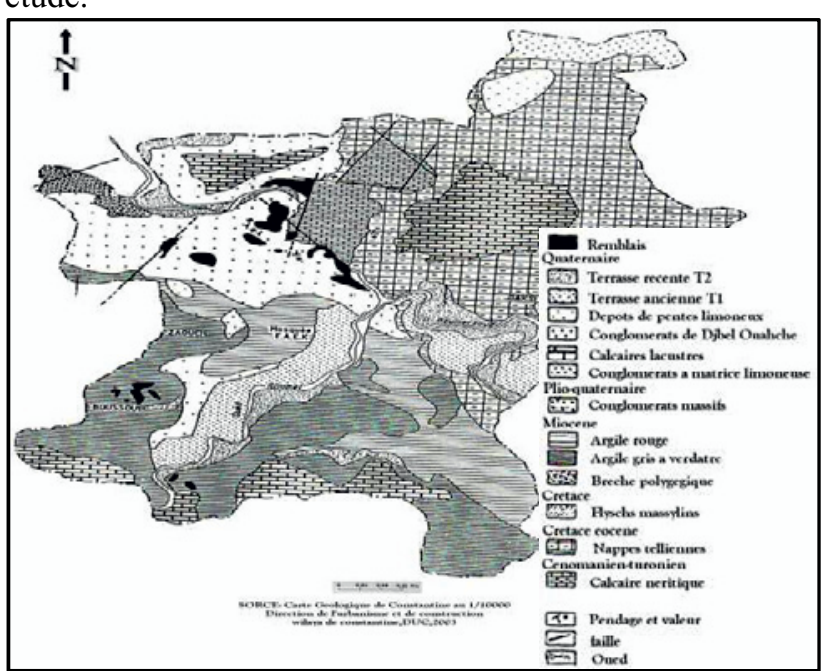

Fig. 2. Carte géologique de la commune de Constantine (Source DUCH 2003). 


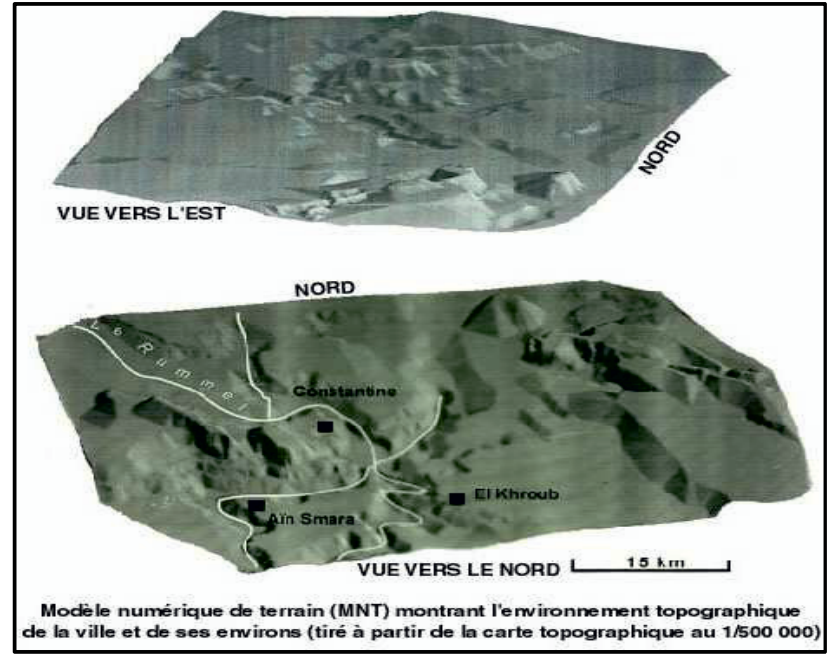

Fig. 3. morphologie de Constantine et de sa région (Belouar 2005) [14].

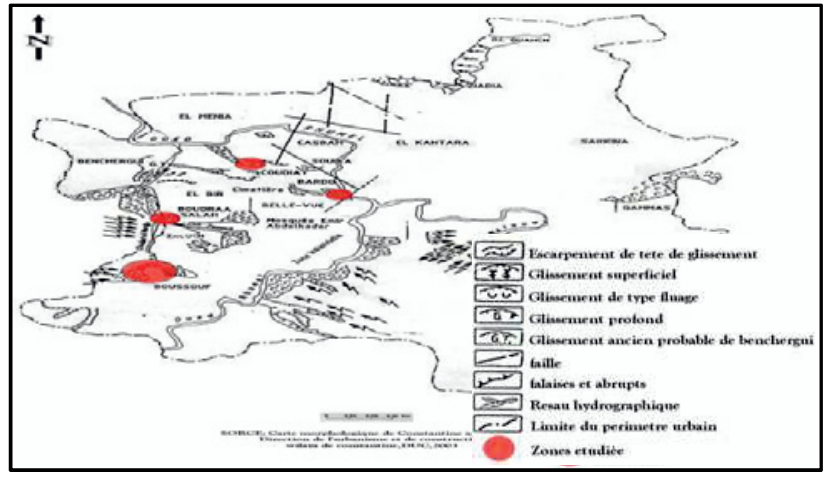

Fig. 4. Carte géomorphologique de la commune de Constantine (Source DUCH 2003) [15].

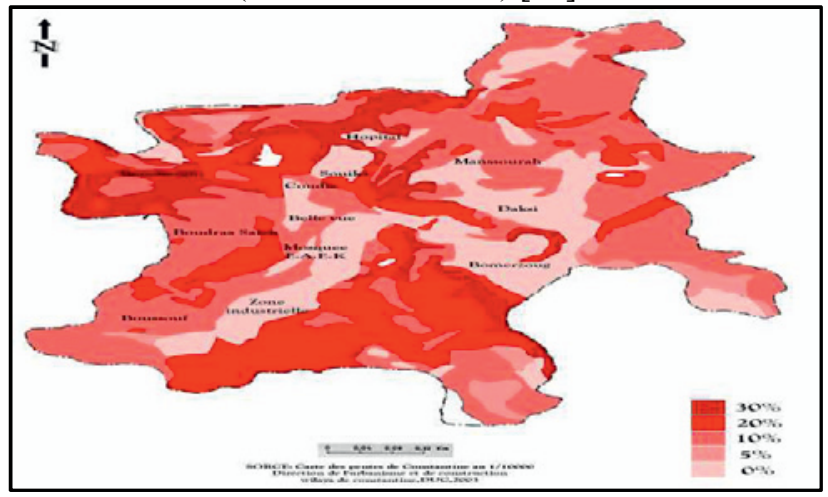

Fig. 5. Carte des pentes de la commune de Constantine [15].

Les risques identifiés sur la région de Constantine sont essentiellement liés à des facteurs structurels tels la géologie, la topographie, la structure ( faillé ou non ), l'hydrologie et les paramètres géotechniques et d'autres aggravants tels que l'action humaine (urbanisation anarchique, excavations en pied des talus, canalisation défaillante des eaux usées ) , les précipitations saisonnières sans pour autant parler de certains paramètres à prendre en compte lorsqu'il s'agit d'un mouvement ancien ou nouveau.

L'analyse de ces facteurs déclenchant dans ces zones va permettre de définir des zones de présomptions dans ces sites ou ces facteurs sont très importants de part la vulnérabilité et les enjeux ( bati et biens et équipements) comme le montre la figure 7 et 8 (c'est le cas d'un seul site à savoir Boussouf).

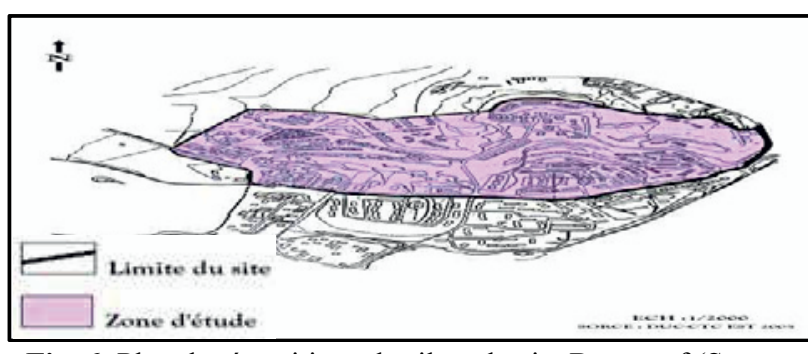

Fig. 6. Plan de répartitions des ilots du site Boussouf (Source DUCH/CTC Est 2005).

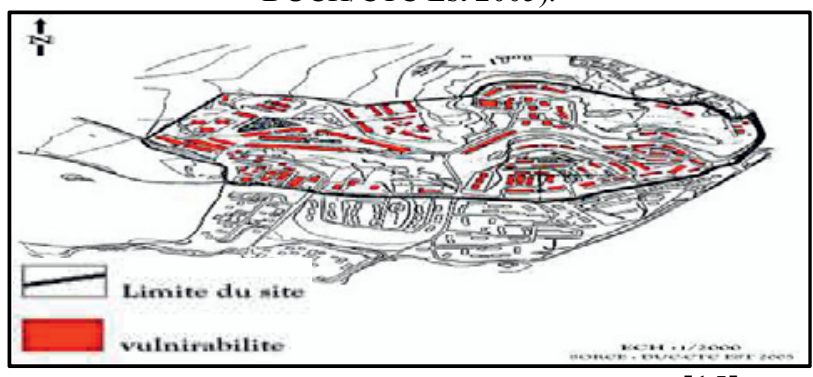

Fig. 7. Plan de vulnérabilité du site de Boussouf [15].

\subsection{Zonage d'une région potentiellement instable}

Le but est la détermination d'une cartographie pour la région pour être utilisée comme document de base joint au plan directeur d'aménagement et au plan d'occupation des sols afin d'éviter les erreurs d'appréciations des versants (Fabre et al 1998) [16].La méthodologie proposée est basée sur une analyse qualitative des facteurs déterminants (Belouar et al 2005) [17-18]. la problématique des mouvements de terrain. Le travail consiste à :

- Inventorié tous les sites de la région touchés par ce phénomène.

- Analyser les différentes études réalisées auparavant sur ces sites.

- Mettre au point les facteurs déterminants selon la compilation des données.

- Dresser les cartes d'aléas et d'enjeux

- Définir l'aléa des mouvements de terrain comme zone d'apparition d'un phénomène sur une région étudiée (carte de zonage).

A partir des données collectées pour chaque zone, mises en forme et inventoriées sur une base de donnée au sein du laboratoire des matériaux et durabilité des constructions de l'Université de Constantinel pour tous les sites de la ville et en étroite collaboration avec le laboratoire de l'unité de recherche du génie civil U.R.G.C de Géotechnique de l'Institut National des Sciences Appliqués I.N.S.A de Lyon on peut illustrer ceci par une représentation cartographique tout en utilisant la superposition des cartes pour l'obtention d'un zonage géotechnique. Extraire les facteurs déterminants par l'analyse des données ; Quantifier la probabilité de l'aléa mouvements de terrain et en cartographier la répartition.

\section{La Carte du zonage}

Ainsi si l'on fait la transposition des données recueillis, inventoriées et mises en forme sur les cartes et en appliquant la méthode d'estimation du risque potentiel 
d'instabilité en un secteur considéré, donne pour le cas choisi, le zonage suivant comme le montre la figure.8. L'examen de la carte montre trois zones .On remarque que les zones qui présentent un risque potentiel ou déclaré sont liées essentiellement au niveau des formations marneuses. Les secteurs susceptibles d'être aménagés à condition de procéder à des travaux de stabilisation du sol, et assurer un mode de fondation qui doit absolument prendre en compte le risque de gonflement. Pour un autre secteur on rencontre des formations argileuses limoneuses sableuses où les risques sont faibles, et leur aménagement ne pose à priori aucune contrainte d'ordre physique.

Ce zonage géotechnique donne une physionomie générale du secteur considéré, qui permet d'éviter les grosses erreurs pratiques. Mais au niveau de l'exécution ponctuelle, l'étude spécifique reste irremplaçable.

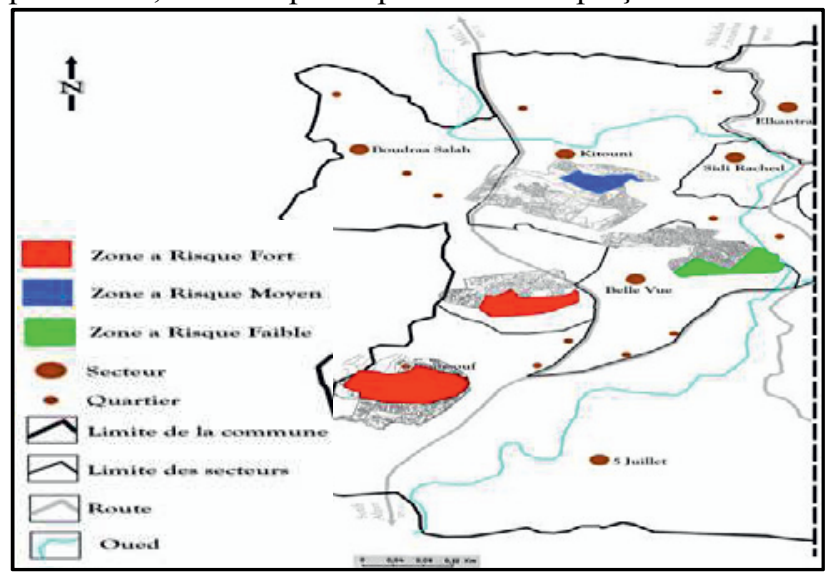

Fig. 8. Carte de zonage géotechnique de quelques sites de Constantine.

\section{Conclusion}

Nous avons, au travers de cet article présenté une approche cartographique qui offre un intérêt particulier pour localiser les zones exposées aux mouvements de terrain et donner une évaluation qualitative du risque.

Le site de la wilaya de Constantine s'est admirablement prêté à la mise au point de la méthode proposée en raison de la bonne connaissance des différents secteurs sujets à des mouvements évolutifs et de l'abondance des informations géologiques, topographiques, hydrogéologiques, géotechnique ainsi que la connaissance du bâti.

Il serait souhaitable d'entreprendre d'autres études plus poussées avec l'utilisation des logiciels tels les SIG et faire une étude comparative avec celle déjà réalisée.

\section{References}

1. J. Vaunat, Gestion du risque et surveillance des zones instables, Conférence Canadienne de Géotechnique, Vancouver, 20-24 (1997).

2. E. Leroi, Landslide hazard - risk maps at different scales, Objectives, tools and developments. Proceedings of the Seventh International Symposium on Landslides, Trondheim, Norway, June 17-21, vol. 1, 35-51 (1996).
3. B. E. Berggren, J. Fallsvik, L. Viberg, Mapping evaluation of landslide risk in Sweden, Landslides Bell ed., 873-878 (1992).

4. P. Ahlberg, L. Viberg, Experience of landslide risk consideration in land use planning in Sweden, Bonnard ed Lausanne, 1091-1096 (1988).

5. E. W. Brand, Landslide risk assesment in HongKong, Bonnard ed Lausanne, 1059-1074 (1988), R. Fell, P. Finlay, G. Mostyn, Framework for assessing the probability of sliding cut slopes. Landslides Senneset ed. 201-208 (1996).

6. P. Canutti, The activity programm on landslides of NRC's research group on hydrogeological catastrophes. Bonnard ed Lausanne, 1127-1130 (1988).

7. W. T. Barros, C. Amaral, R. N. D’Orsi, Landslide succeptibility map of Rio de Janeiro, Landslides Bell ed., 869-871 (1992).

8. M. S. Rosenbaum, M. E. Popescu, Using a geographical information system to record and assess landslides related risks in Romania, Landslides Senneset ed., 363-370 (1996).

9. S. Paulsen, et all, Rapport d'expertise sur les glissements de terrain de la ville de Constantine (Algérie), Institut Fédéral des Géosciences et des ressources naturelles Hanovre (R.F.A), $\mathrm{N}^{\circ}$ arch. 117989 (1999).

10. A. Belouar, A. Benaissa, A. Seridi, Stratégie et Prévention des Risques Naturels. Cas de la ville de Constantine (Algérie), Séminaire International sur les Risques Naturels liés aux Glissements de Terrain et d'Eboulement Rocheux. Guelma (Algérie) (2005).

11. P. Deleau, Etude stratigraphique de Constantine (Djebel Oum Settas).These de doctorat, pub serv. Carte géol Algerie,(2), $\mathrm{N}^{\circ} 14$, texte et atlas, (1952).

12. J. M. Villa, Carte géologique à 1/50 000 El Aria avec notice explicative, Publ. SGA, (1977).

13. Lahondere, Les séries ultra - telliens d'Algérie Nord orientale et les formations environnantes dans leur cadres structurales, Thèse d'état U.P. Sabatier Toulouse, (1987).

14. A. Belouar, N. Mongereau, L. Vinet, A. Seridi, Description géologique et géotechnique de quelques glissements de terrain. Cas de la ville de Constantine (Algérie) Proceedings of the International Symposium on Urban Geotechnics. Lille- France, (2005).

15. DUCH : Direction de l'Urbaniseme de Constuction et Habitat $(2003,2005)$.

16. R. Fabre, B. Clément, Th. Lebourg, Les mouvements de terrain en Gironde (33), Cartes de risque et d'aléa. XVI ème Congrès Universitaire de Génie Civil, Reims, Vol. 2, 380-387 (1998).

17. A. Belouar, Détermination des caractéristiques géotechniques d'une zone potentiellement instable. International Conférence on Geotechnical Engineering, Beyrouth, Lebanon, 637-642 (2004).

18. A. Belouar, Analyse et Valorisation des Caractéristiques Géotechniques des Sols Fins, Colloque International Sols et Matériaux à Problèmes, Tunisie, 124-136 (2007). 